\title{
Book Review: Psychoanalysis and Digital Culture - Audiences, Social Media, and Big Data by Jacob Johanssen \& Event Hori- zon - Sexuality, Politics, Online Culture, and the Limits of Capitalism by Bonni Rambatan \& Jacob Johanssen
}

\author{
Jamie Ranger \\ St. Hugh's College, University of Oxford, Oxford, UK, \\ james.ranger@st-hughs.ox.ac.uk jayranger@hotmail.co.uk
}

\begin{abstract}
Jamie Ranger reviews Jacob Johanssen's Psychoanalysis and Digital Culture - Audiences, Social Media, and Big Data' The book offers a comprehensive account of our contemporary media environment - digital culture and audiences in particular - by drawing on psychoanalysis and media studies frameworks. Event Horizon, written by Johanssen in co-authorship with Bonni Rambatan, applies a psychoanalytic lens to online culture, modern sexuality and politics to examine the functioning of capitalist ideology.
\end{abstract}

Keywords: digital culture, affect theory, affective labour, social media, big data

\section{Book Overview: Psychoanalysis and Digital Culture}

In the Introduction, Johanssen presents an overview of the relationship between psychoanalytic theory and media studies, suggesting that a return to concerns about subjectivity has brought the former back in from the proverbial cold. Although media studies and communication theory often seek to analyse a network of rational actors, psychoanalysis "presents a notion of subjectivity that includes rational, conscious elements but also includes irrational elements and the unconscious" (5). Furthermore, there is overlap between theories of media subjectivity and the notion of the transitional object in psychoanalysis, where the latter refers to objects that allow for the subject to feel secure and sensual environment (6). Johanssen argues that his own work can be understood as one of many in the growing field of "psychoanalytic media studies" (8), and that the application of affect theory to media is appropriate because in the former the body is treated as an "interface". Specifically, " ust as affect is, ontologically, about a processual relationality, the interface is, broadly speaking, a facilitator or enabling moment of a state of in-betweenness" (9).

In Chapter 1, "Audiences, Affect, and the Unconscious", Johanssen argues that by utilising the Freudian account of affect, responses by media users can be understood at both conscious and unconscious levels. Johanssen concretises the "safe spaces" that are described in Anzieu's theory of affect via the skin ego as the media consumed by a given set of users. In the context of reality television show Embarrassing Bodies, the thought goes that close-ups of surgeries, flesh lacerations or abnormalities may provoke a visceral reaction that can be observed, which will then be later rationalised by the media user to whom it has occurred. The theoretical contention is that "affects are bodily sensations that are experienced and brought into consciousness or can be repressed altogether" (29). Furthermore, "affect always has a psycho-biographical element to it and so affective experiences always need to be seen in relation to a 
subject's conscious and unconscious history. Of course, the memory as such is not reemerging, for memory is always subject to variation and change" (34).

The chapter concludes by listing Anzieu's eight functions of the skin ego: holding, where the baby forms a mental image of the mother's touch that both in fantasy and reality creates a sense of security; containing, touch and sound (voice) that either enwraps the baby in security, or if absent, creates anxiety; thirdly, protection against stimuli, fourthly, the skin ego individuates the self, giving the baby a sense of their uniqueness, a precursor to personhood; fifthly, intersensorality, in that the touch of the mother becomes a sensorial reference point; sixthly, supporting sexual excitation, in that the baby's skin experiences are pleasurable (kissing, bathing, holding, feeding), which is a precursor to embodied sexual experience; libidinal recharging, in that internal affective tensions are maintained in balance; finally, registering traces, in that the skin provides information about the external world (40-41).

In Chapter 2, "Affect, Biography, and Watching Reality Television", Johanssen draws upon interviews conducted with media consumers of the TV show Embarrassing Bodies, the premise of which is that individuals with rare and unusual bodily issues present them to one of the doctors (and therefore, national television) to receive treatment. The interviewees describe the doctors as maternal and containing figures, with the same doctor-presenters used in each episode to convey a sense of stability and familiarity for the audience. Interviewees refer to the show as "entertaining, then did not provide more clarifications on the topic and described the show in different terms ('fascinating', 'interesting', 'unbelievable') and then implicitly returned to the theme and noted that it felt 'wrong'...or 'sick'" (58). Johanssen explores these tensions in their discourse through the lens of voyeurism, suggesting that "voyeurism is an on-going process without end [...] [it] is not only about affect and entertainment but also about repetitive viewing to achieve mastery over bodily states" (60-61). The TV show may be interpreted as a substitute for the experience of bodily trauma in the interviewees, in that the screen "provides a space for subjects to (unconsciously) project aspects of their biographies on" (63). Voyeurism in this context, therefore, is neither neurotic nor damaging for the subject's well-being, and instead Johanssen understands it as a means by which the interviewees encountered their inner experience within comfortable distance (63). The programme itself cultivates "a feeling of being in control and learning about medicine" (64). Johanssen concludes that the affective moments witnessed in the interviewees demonstrates the theoretical conceptualisation of the viewing experience as embodied, in that "the ego is always a bodily ego" (67), and "from a psychoanalytic viewpoint, an apparent desire to be contained constituted a key moment that also marks the (unconscious) appeal of Embarrassing Bodies" (68).

In Chapter 3 "Unable to Tweet: Inhibition and the Compulsion to Share", Johanssen argues that "the programme resulted in such affective responses to the show in the interviewees that they may have been unable to engage with it on social media" (70). Many interviewees confessed anxieties, insecurities and traumas associated with their body and claimed these experiences were unrelated to their enjoyment of the programme. Johanssen suggests that disclosing these experiences during the interview process was facilitated by the programme without conscious awareness (70). Johanssen undertakes a brief literature review of psychological approaches to social media, suggesting that contemporary platform users are inculcated into sharing to demonstrate their social value or status and that they share in anticipate of an other's judgement. Studies acknowledge that users are often aware of legal, privacy and ethical issues surrounding their social media use and thus imply that social media sharing is "a conscious process guided by rationality and reason" (75). The suggestion is that 
due to the psychological impact behind the appeal of Embarrassing Bodies, the show is not conducive to the forms of public disclosure that social media thrives upon encouraging. Johanssen explains that viewers who otherwise posted regularly on social media, and even followed the profiles of the featured doctors, did not post about the show, despite Channel 4 branding the show with social media in mind and the platform's in-built injunction to discuss it. More specifically, the interviewees were prone to "affective discharges" when describing their viewing experience, with wordless phrases such as "eurgh", "eeeh" and "eeuuh" appearing in Johanssen's transcripts (92). Interviewees experienced a form of entertaining voyeurism but found it difficult to articulate why on the conscious level, leading to the conclusion that "affective memories relate to memories that are in relation to their bodies" (92), hence the affective discharge of noises rather than the clearer application of descriptive or evaluative language.

In Chapter 4, "Affective Labour and the Body: Theoretical Developments", Johanssen discusses the "the increasing focus of data in the realm of the digital and how psychoanalysis may help to make sense of it" (94). Johanssen enters the debate surrounding immaterial and affective labour in the literature, immaterial labour is broadly characterised as the extension of commodification into activities that exist beyond our traditional understanding of "work", including communication as a provider of information. It is often criticised for suggesting that the information age has heralded a new and distinct form of exploitation when Marxist feminist scholars have previously discussed the idea of unpaid labour in domestic, care and service work. Nevertheless, the optimism that underlies Hardt \& Negri's concept of immaterial labour is the potential marshalling of affective labour towards emancipatory ends, where "affect becomes a power of transformation through and towards the commons" (100).

Johanssen argues nonetheless that the former configuration "may be convincing in relation to changing labour practices but is often unclear and dualistic" (105). He concludes that given the growing body of literature that draws upon "affect" to underscore the psychological and sociological components of digital labour, there is scope for the more specific conceptualisations of the term as applied in psychoanalysis.

In Chapter 5, "Affective Labour on Social Media", Johanssen considers the social media use of people with facial disfigurements to inform the debate surrounding embodied and affective approaches to digital labour. Johanssen approaches these "engagements with social media as labour, rather than mere use, self-representation, affordance, or online activity [...] because the interviewees implicitly spoke about their self-representation in a vocabulary that was about labour and alluded to notions of entrepreneurialism, affect, self-surveillance and self-branding" (109). Johanssen draws upon the recorded narratives of the participants in the study to mask the case for the "skin ego" approach to social media. Specifically, as social media provided a space for engaging with their own subjectivities and other users, the platform may have "contributed to a sense of security and feelings of containment for users in the first place" (115). However, participants also described social media platforms ambivalently, concerned about the prospect of being exposed or put upon by a perceived swarm of judgemental fellow users, and these anxieties influenced how and what they posted. Johanssen considers this an act of "affective labour because it ultimately seeks to create the perfect product that both the users as well as others on social networking sites are pleased with" (115). Drawing on the research that focuses on the neoliberal subjectivity of social media, Johanssen argues that "subjects not only bring themselves to the labour market online, but they also generate their own advertising campaigns that can be seen on social media and personal websites in order to be noticed and acknowledged by other users" (117). Johansson observes that despite their general 
acknowledgement of the exploitative relationship between platforms and users (124), the indifference of participants may be explained by the fact that "affective labour on social media may be empowering because it gives the individuals with bodily differences a voice and it challenges ideological and hegemonic appearance standards and stereotypes online" (130). Although neoliberal principles objectify the subject and reproduce conditions of affective digital labour exploitation, "acts of self-representation are very important because they disrupt social media hegemonies" (138).

In Chapter 6, "The Perverse Logic of Big Data", Johanssen provides an account of the varying approaches to data mining in the media and communications literature and argues for an approach that goes beyond technical processes and includes "questions of subjectivity, power, and autonomy" (143). "Big data" is defined as "both a description of the effort and mechanism itself through which to gather, extract, process, and analyse (digital) data or create such data in the first place through the conversion of other analogue data into the digital format" (141-142). Johanssen suggests that "data mining and psychoanalysis are both driven by a desire to know the other and to accumulate data. To some degree, the analyst and patient together extract data from the patient's (un)conscious life experiences and interpret them and use them in some way" (145146). Big Data processes are interpreted by psychoanalytic theory as perversions, in that "the perverse subject, or pervert, regards the other in a relationship as an object" (148) and platforms lure media users "under the guise of communication and connection", only to exploit them by translating them "into data profiles to advertisers" (148). Johanssen concludes that "social media are dependent on individuals who create and use data, but a real meaning and economic asset is only acquired through an accumulation into large data sets" (165). However, platform users are lured onto social media because of its promise of quick, easy satisfaction, and therefore "the relationship between us as subjects and the services and platforms which mine/use our data is complicated and symbiotic" (165). Nevertheless, the thought is that "if so much of the content we produce online and of other data that are gathered along the way can be used in ways we may anticipate but do not know about, subjectivities in relation to the digital, then, are characterised by a sense of paranoia and uncertainty" (167).

In the Conclusion, Johanssen provides further thoughts on affect and digital media. Johanssen contends that "affective responses that do not seem to have an origin or explanation that can be pinpointed or known (as many interviewees have discussed) may thus be aroused because of (unconscious) memory traces that relate to past affective experiences" (171). Furthermore, in the context of media saturation in our culture, media "are distinctly affect triggering" (172). Returning to Anzieu's psychoanalytic toolkit, Johanssen argues that the idea of the skin envelope is useful for thinking through media and audiences because it focuses on the virtual and material qualities of skin, protecting against external stimuli and unconscious affects, memories or thought that cannot pierce through the internal layer and be externalised (175-176). Skin holds across our bodies, but is always broken and fragile, with each episode "a possibility of mastery and a final containment presents itself" just as "the skin ego designates a phase that is characterised by the growing baby who - we could say - unconsciously and consciously awaits the ego and a degree of separation from the mother to gain more independence" (178). To apply the analogy to social media, the relationship between platforms and users may be characterised as symbiotic yet fragile: we anticipate how the platforms might use the data we provide, they use algorithms to persuade and entice us into new avenues of attention: "the digital subject [...] constructs and reconstructs herself and is constructed and reconstructed by others whereby past, present, and future elements are merged into a dynamic, floating 
subjectivity" (180). It is this psychological proximity between subjects and the media services and technology they use that justifies the incorporation of psychoanalytic theory into media studies (181).

\section{Book Overview: Event Horizon}

In the Introduction, "Into the Digital Black Hole", the reader is introduced to the concept of the Event Horizon, the point of no return on the outermost region of a black hole beyond which nothing, including light, can escape. The inevitability of the Event Horizon is likened to contemporary capitalist subjectivity, compelled by an unstoppable force towards a place of technological singularity. There is also a play on its usage by philosopher Alain Badiou, where an Event is an ontological condition by which new subjectivities are produced. The most powerful analogy is with the movie Event Horizon, in which the titular spaceship is revealed through quirks of science-fiction to be mysteriously gaining consciousness, horrifying - and eventually possessing - the crewmembers. The thesis of the text is that Lacanian psychoanalytic concepts may be applied to our contemporary digital landscape to better understand the modern psychosocial conditions of capitalism (Rambatan \& Johanssen 2021, 6).

Chapter One, "Broken Circles, Infinite Loops", undertakes an analysis of conspiratorial thinking and post-truth in the context of tech firms. The authors proceed with one of the most clearly written descriptions of Lacan's discourses I have ever read. The enjoyment is made that "the enjoyment present in post-truth thinking is twofold" (21), in that there is paranoiac enjoyment, "the thrill of imagining evil cabals and covert propaganda" alongside narcissistic enjoyment, "the pleasure of being among those who have woken up to the truth" (21). The authors suggest that by inverting the formula of Lacan's fifth discourse of Capitalism, we see Conspiracy as its mirror image. Furthermore, the argument is made that the progress of capitalism can be linked to the project of expanding its knowledge of the Subject's desire (25). Drawing on contemporary interpretations of Lacan's discourses, the chapter concludes that "it is becoming increasingly imperative to affirm our subjectivity as a structural negativity that will forever resist the totalising vitalist fantasy of capitalism" (32).

In Chapter Two, "Networks and Psyches: Unleashed and Restrained", in the context of the social media literature, it is proposed that technology firms play with our desire through the ideological promise of attention, doing so through a "dis/individualising operation" (37). The operation works as follows: "social media speak to us as subjects on a deep individual level as we are encouraged to realise ourselves within our online personas" whilst also disindividuating us by reducing us "into data to be commodified" (37). The claim is that "today's capitalism functions on the valorisation of antagonism. The hatred of the different other is the engine of profit creation for the ruling class. For all their promise of authenticity and innovation, social media and tech companies require a society to keep being divided and in conflict" (39). With this analytic in mind, online harms such as trolling must be "situated as part of a wider, semi-permanent atmosphere of outrage, offence, and heightened affect" (43). Internet culture and its dominant platforms, place the subject in a superposition between psychosis and perversion - "dis/inhibited networks have fundamentally altered our way of reading, our very gaze" (51).

In Chapter Three, "The Cute Subject", the authors reflect on kawaii culture in digital spaces. The authors contend that "the obsession for cuteness is vicious: it is not merely a passive search of childlike helplessness to elicit a feeling of warmth, but an active movement of mutilating the object, enforcing its pitiable position" (54). The claim is that memes are the pictographic means by which individuals are translated on social media 
into cute subjects, for memes embody the ironic circulation of contemporary online culture. Memes contribute to the "underlying psychodynamic of habit, repetition, and minimal alteration that fits perfectly with the clustering patterns of network culture" (65). Drawing on Freud, the authors argue that selfies may be understood as forms of exhibitionism that reveal the vulnerability of the subject, "exposing a naked hunger for approval and the desire of the Other" (70). It is through the transmission of cuteness we sustain the fantasy of being seen by the Other, but social media also brings entrapment in systems of platforms, selfies, memes, likes, comments, and shares that we come to loathe. The contention is that trolls "hijack the language of cuteness - memes, but also fake selfies - and the fundamental vulnerability that comes with it, and present this fact right up to our faces: authenticity is powerless, empathy is futile" (72).

In Chapter Four, "Heteropessimism and Digital Anesthesia", the authors unsurprisingly reveal the link between the psychotic/perverted activities of trolling, and the problematical framing of sexuality in online micro-communities. The most prominent and notorious example are Incels (short for involuntary celibates), a community of young predominantly White - men, who believe that their life prospects have been undermined by women and feminism (81). Incels acknowledge the supposed impossibility of acquiring sexual partners, "yet nonetheless define themselves via a lamentation of and defence of this fact" (82). Incels are considered symptomatic of a broader heteropessimism, where straight relationships are framed as increasingly inaccessible hopeless, and often comes with an "idealisation of non-straight experience and relationalities" (84). By framing sexual culture on social media this way, the authors contend that both "feminism and anti-feminists are thus united in uncertainty and caution about how to live, have sex, and love together" (85). The reliability of platforms, the frictionless of online experiences, are juxstaposed with the embodied fragility and messiness of human sexuality: "nonsensical articulations exploded into the scene as the Real returns with a vengeance: our bodies, in the end, are always unreliable" (97).

In Chapter Five, "Playful Perversions: Narcissism and the Gamification of Control", the authors demonstrate that despite the claim of capitalism to a total understanding of our individual and collective desires, "capitalism works on a fundamental misrecognition of our desires" (104). Big data thrives on the accumulation of information regarding our social media profiles, tracking our choices, and our histories of consumption and attention, but we are more than our online avatars: "it is clear that capitalism wants us to be a subject reducible to its specular image" (113). By fleshing out Byung-Chul Han's contentions regarding psychopolitics, the authors provide an account of the narcissistic subject that capitalism encourages, and offers a plausible, structural explanation for the absence of digital solidarity, including amongst fellow ideological travellers.

In Chapter Six, "Too Close, but not Close Enough: Politics and Sexuality in Times of the Alt-Right", we are introduced to the extreme example of the compelled technosocial subject in Erik C. Estavillo, an American citizen who compulsively sues any technology companies that interfere with its unfettered access to their computer networks, even when banned from the PlayStation network for foul and abusive language during gaming sessions. Estavillo positions himself as a victim against the sheer addictiveness of technology products, an eccentric figure who believed all too readily in the promise of living out one's fantasies in a sanitised cyberspace without consequence. Through a reading of Tinder logic, the authors conclude that "our political situation today can also be read as a reactionary politics against creepiness" (138), where our systems of transparency and surveillance have become so ubiquitous and accessible that it is only our own dignity that prohibits us from becoming uncomfortably familiar with strangers through digital parasocial arrangements. Political optimism is found in 
the Black Lives Matter movement, precisely because it resists the "temptation of cute displays of multicultural identities where diversity is treated as a sanitised collection of text and images" (140).

In the Conclusion, "Event Horizon", the authors argue that "today life is seen not only as a tool of production but as a mine for data production that can generate more wealth which the majority of the world will be unable to access" (142). There is a political argument for the necessity of reconfiguring our digital technologies - "a topological movement, a reorganisation of the ways Subjects access their jouissance" (144) - a turn away from the frictionless, sanitised cyberspaces and instead towards an acknowledgement of materiality, of our own embodied fragility and the necessity of shared networks of care and solidarity. The authors leave us with a reflective question: "how will we live when our digital social spaces are not defined by rage against the Other but by playful creative interactions which serve as our own means of production, where facilities for the common good are free and participation in the social fabric brings us wealth?" (154).

\section{Reflections}

The first book draws upon the predominantly clinical theoretical insights of psychoanalysis to develop an empirical investigation into the conscious and unconscious engagements of media users. Johannsen's primary psychoanalytic influence is Didier Anzieu, specifically his notion of the "skin-ego". Johanssen explains that "Anzieu conceptualised the beginning of a subject's life as characterised by the relational unity of mother (or caregiver) and baby (and father and others to a lesser degree)" (Johanssen 2021, 3).

The overarching theoretical premise for the book moving forward is that "any engagement with media occurs at the virtual level (through the very consumption of something mediated) and the affective level (through being affected by it bodily)" and so "media use is related to fantasy, thought, and utterances as well as to bodily responses that are situated at the intersections of consciousness and the unconscious" (3).

Johanssen's lone project is an academic monograph that aims at demonstrating the value of psychoanalytic concepts to those interested in interpreting audience data, especially in the context of new media and overlapping media discourses. It is a welcome intervention into the debates surrounding the applications of affect theory to digital contexts and would be an illuminating read for anyone interested in working with critical accounts of affect and the increasingly proximate relationship between subject and media.

By contrast, Rambatan and Johanssen's joint work is aimed at a more general audience, pitching Lacanian psychoanalysis to a readership perhaps vaguely familiar with exponents such as Slavoj Žižek, and the niches of the Internet that are utilised as case studies. The latter is more political and polemical, with an authoritative voice and a reassuringly vast knowledge of the hyper-specific discourses that increasingly permeate Internet platforms and online sub-cultures. The book is important for understanding the rise of the Alt-Right, the distinctive psychological components of Internet culture that new fascist groups exploit, and the perverse tendencies of social media platforms that transcend political orientation and must be accommodated by any prospective emancipatory Left project.

If the overall aim of the two texts is to demonstrate the viability and value of psychoanalysis to understanding our contemporary conjuncture, then I argue both are successful in fulfilling such a task. The descriptions of psychoanalytic concepts are clear 
and incisive, and their cultural readings feel intuitively plausible alongside the richly drawn examples (in the case of the author's reproduction of McCloud's picture plane, quite literally) (Rambatan \& Johanssen 2021, 63)! For those readers agnostic to the incorporation of psychoanalysis into media and communication studies and political analysis, these texts serve both as sharp introductions as well as persuasive and timely applications.

\section{References}

Johanssen, Jacob. 2021. Psychoanalysis and Digital Culture: Audiences, Social Media, and Big Data. London: Routledge.

Rambatan, Bonni and Jacob Johanssen.2021. Event Horizon: Sexuality, Politics, Online Culture, and the Limits of Capitalism. Winchester: Zero Books.

\section{About the Author}

Jamie Ranger

Jamie Ranger is a doctoral candidate at St. Hugh's College, University of Oxford in the Department of Politics \& IR. He writes on the politics of speed, technics, media, space and radical democratic theory. He tweets from @jamieandhisego. 